\title{
Role of carbon addition on the microstructure and mechanical properties of cemented tungsten carbide and steel bilayer
}

\begin{abstract}
The development of several novel multifunctional components to perform specific unique functions is directed towards meeting the demands for advanced components in industries. In this study, the role played by carbon ( $\mathrm{Cgr}$ ) variation on the steel part composition of cemented tungsten carbide and steel bilayer processed via powder metallurgy was investigated. Microstructural examination through field emission scanning electron microscopy (FE-SEM) and energy dispersive spectroscopy (EDS) revealed the presence of detrimental eta carbide phase (M6C) distributed across the interface of sintered bilayer compacts. A significant reduction of M6C was observed with $0.8 \mathrm{wt} . \% \mathrm{Cgr}$ when interlayer diffusion was accelerated resulting in better morphology and higher hardness values of 735.70 and $150.97 \mathrm{kgf} \mathrm{mm}^{-2}$ in $\mathrm{WC}$ and Fe layers, respectively. Tensile strength property was evaluated to examine the sintering compatibility and the interfacial bond strength of bilayer specimens. Excellent bond strength was achieved in all sintered bilayer with increasing $\mathrm{Cgr}$ level and enhanced densification which consequently improved tensile strength by $19 \%$.
\end{abstract}

Keyword: Bilayer interface; Carbon content; Eta phase; Hardness; Tensile strength 